Revue Interventions économiques

Papers in Political Economy

48 | 2013

Médiations ou intermédiations dans les dynamiques méta-organisationnelles

\title{
La méta-organisation territorialisée, moteur d'apprentissages collectifs
}

Territorialized Meta-Organization, the Engine of Collective Learning

Martine Gadille, Diane-Gabrielle Tremblay et Antoine Vion

\section{CpenEdition}

Journals

Édition électronique

URL : http://journals.openedition.org/interventionseconomiques/2156

DOI : 10.4000/interventionseconomiques.2156

ISBN : 1710-7377

ISSN : $1710-7377$

Éditeur

Association d'Économie Politique

\section{Référence électronique}

Martine Gadille, Diane-Gabrielle Tremblay et Antoine Vion, « La méta-organisation territorialisée, moteur d'apprentissages collectifs », Revue Interventions économiques [En ligne], 48 | 2013, mis en ligne le 01 novembre 2013, consulté le 22 septembre 2020. URL : http://journals.openedition.org/

interventionseconomiques/2156 ; DOI : https://doi.org/10.4000/interventionseconomiques.2156

Ce document a été généré automatiquement le 22 septembre 2020.

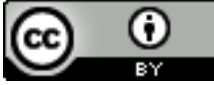

Les contenus de la revue Interventions économiques sont mis à disposition selon les termes de la Licence Creative Commons Attribution 4.0 International. 


\title{
La méta-organisation territorialisée, moteur d'apprentissages collectifs
}

\author{
Territorialized Meta-Organization, the Engine of Collective Learning \\ Martine Gadille, Diane-Gabrielle Tremblay et Antoine Vion
}

\section{Introduction}

1 La réflexion sur le phénomène de construction de méta-organisations a connu ces dernières années d'importants développements en théorie des organisations, tant en Amérique du Nord qu'en Europe. Mais qu'en est-il du poids des contextes sociaux régionaux et de l'enracinement local dans ces processus de regroupement d'organisations? Depuis la vague des travaux sur le type particulier des districts industriels (Bagnasco 1977, Brusco 1982, Benko et Dunford 1991, Pyke et Sengenberger 1992), un pan de l'économie des organisations s'est intéressée à la construction des nouveaux modèles organisationnels propres à l'innovation localisée, qu'il s'agisse des clusters industriels (Saxenian 1994, Brail et Gertler 1999, Brenner 2005, Robinson et al. 2007, Britton et al. 2009, Delgado et al. 2010, Glaeser et al. 2010) ou des plates-formes technologiques (Lemarié et al. 2001, Zeller 2001, Aggeri et al. 2007).

Dans ce domaine, pourtant, la réflexion s'est rarement inscrite dans la problématique de la méta-organisation. La majeure partie des travaux a insisté sur la dynamique inter-organisationnelle, que ce soit pour expliquer les phénomènes d'agglomération, comme dans les études de clusters, ou pour caractériser les formes de coopération, notamment dans les analyses structurales de réseaux. La coopération est ainsi expliquée par ce qu'Emmanuel Lazéga a appelé les rationalités sociales d'entrepreneurs interdépendants (Lazéga 2009), et les logiques entrepreneuriales par la capacité à combler un trou structural entre deux réseaux préexistants (Burt, 1992; Deroïan, Steyer, Zimmermann, 2003). Au mieux, la question de la gouvernance des réseaux estelle posée (Ehlinger et al. 2007), mais l'accent mis sur les dimensions stratégiques prend trop peu en compte la dimension cognitive des médiations ou des intermédiations. Les intermédiaires peuvent certes agir parfois seulement comme pourvoyeurs de 
ressources, au sens de l'analyse de réseaux, ou comme des traducteurs dans une chaîne relationnelle, au sens de Michel Callon (1986). Cependant, il arrive aussi qu'ils se révèlent comme de véritables " intégrateurs cognitifs ", c'est-à-dire des agents capables de réduire des incertitudes cognitives en instituant de la coordination par des artefacts (Latour, 2001) spécifiquement dédiés à la création d'opportunités de collaboration entre organisations parties prenantes. La responsabilité de la conception et de ces artefacts qu'ils soient de l'ordre du simple brainstorming (Conein 2004), de la transformation d'idées en projets, ou enfin de la conduite de projets à la fois transdisciplinaires (Gibbons et al. 1994) et ancrés dans la localité, incombe à un métaorganisateur qui mobilise des liens de subordination salariale, une structure de gouvernance et des moyens financiers publics et privés a cette fin

3 La question des conditions de réussite du point de vue des acteurs parties prenantes et plus largement des territoires régionaux de ces formes de médiation ou d'intermédiation sont au cœur des contributions du présent numéro. La publication de ces articles est pour nous l'occasion d'éclairer des recherches récentes renouvelant, à partir d'un travail sur le concept de méta-organisation, la notion de gouvernance territorialisée. Pour introduire le débat, et préciser l'apport de ce numéro, nous introduisons la notion de méta-organisation et argumentons que si l'importance des règles et du lien entre coûts et structures reste au fondement des approches néoinstitutionnalistes, celles-ci présentent des limites dans la conceptualisation de la méta-organisation. Nous insistons ensuite sur la nécessité de réintroduire la dimension territorialisée des apprentissages, et soulignons enfin la position délicate du métaorganisateur, présenté comme un « maître de contexte » fragile.

\section{La méta-organisation dans une perspective néo- institutionnaliste : un point limite}

4 La notion de méta-organisation vise à comprendre les processus accrus de regroupement d'organisations selon des structures diverses de nœuds de réseaux ou de fédérations assurant un rôle organisationnel de "secrétariat" selon la formule de Göran Arhne et Nils Brunsson (2004). Hormis le lien de subordination impliqué par les contrats salariaux qui vont permettre de spécifier des ressources propres à la fonctionnalisation du secrétariat, les formes d'intégration des adhérents ou membres sous forme de personnes morales peuvent varier, mais ne sont elles-mêmes pas définies par une relation verticale ou un ensemble de contrats formels de coopération. Elles laissent de surcroît à chacun une certaine autonomie d'action dans la poursuite du but commun. Pour ces adhérents ou personnes morales membres, des droits et devoirs réciproques se substituent aux liens de (quasi)-subordination formels. Chacun garde $a$ priori sa spécificité, mais s'engage à mutualiser des ressources et à participer aux initiatives sans y être fortement contraint par une autorité surplombante.

5 Si la notion de méta-organisation est bien présente dans la perspective néoinstitutionnaliste, elle constitue d'une manière une sorte de point limite de ces théories, notamment sur les deux dimensions classiques de ce courant que sont la forme des règles et le lien entre coûts et structures.

6 Sur la question des règles, l'ouvrage de Jeffrey Pfeffer et Gerald R. Salancik (1978) sur le contrôle externe des organisations a, on le sait, constitué un tournant dans la théorie 
des organisations, en rompant avec la logique de maximisation des ressources dans la compétition, et en insistant sur la force des contextes sociaux, des règles et des institutions pour les organisations. Cette perspective désormais classique de l'importance des règles dans l'accès aux ressources constitue un premier acquis de la réflexion sur la construction de la méta-organisation.

Les travaux de Arnhe et Brunsson (2004) ont particulièrement insisté sur l'importance $\mathrm{du}$ droit mou (soft law) et des règles volontaires dans la construction des alliances, consortiums, et autres regroupements. Les raisons de cette préférence pour le droit mou (ou souple) sont à la fois procédurales, instrumentales et statutaires. Elles sont procédurales parce que les règles à la définition desquelles on a participé, et qui ne sont que proposées, suscitent moins de résistance et sont finalement plus facilement adoptées (Dumez 2009). Elles sont instrumentales parce que ce type de règles facilite la collaboration et la coordination, qui est l'enjeu essentiel du rapprochement (Evans et Worth 2005). Une des sources d'efficacité de la méta-organisation est ainsi pour Arnhe et Brunsson (2010) la continuité du travail de rédaction de ces règles et la légitimité du travail d'expertise exercé par une structure de permanents, qu'ils appellent le " secrétariat ». Elles sont enfin statutaires, parce que l'adoption volontaire de ces règles ou normes confère aux organisations le pouvoir d'appartenir à une forme d'activité monopolisée. C'est ici que se situe le point limite de la perspective néoinstitutionnaliste, puisqu'elle retrouve des problématiques classiques de sciences sociales qui assument plus clairement la question théorique du degré de monopolisation des activités. Dans cette tradition, la fermeture vers l'extérieur que constitue l'adoption de règles propres est le moteur du processus de monopolisation, qu'il s'agisse du capital économique (Weber, 2000; Robertson, 2006), des droits à exercer une activité (Sarfati-Larsson, 1978), ou à produire des formats d'objet (Dudouet et al., 2006). Cela ne signifie pas nécessairement que la méta-organisation construit un monopole sur un marché global, mais qu'elle met en place les conditions d'une segmentation typique de processus de concurrence monopolistique au sens de Chamberlin (1933). Parmi les facteurs de monopolisation, Chamberlin faisait d'ailleurs entrer en ligne de compte la localisation de l'activité, aux côtés de la marque et de la qualité. Qu'il s'agisse des pôles de compétitivité en France, de plates-formes technologiques, de consortiums, etc., la capacité du secrétariat à construire des normes techniques et des normes de qualité et à organiser les conditions de sélection des acteurs aptes à produire selon ces normes pose une question qui dépasse l'approche formelle de l'organisation. Cette question est celle du pouvoir économique de la métaorganisation.

8 La mise à l'épreuve des approches néo-institutionnalistes est peut-être encore plus poussée sur la question des liens entre coûts et structures. La réflexion avancée sur le lien entre coûts et structures a été ouverte par Oliver Williamson (1983), lorsque, formalisant la perspective de Ronald Coase (1937) sur l'indétermination et la contingence des capacités de management sur le coût de l'intégration, il mit l'accent sur la détermination des structures de gouvernance par les structures de coûts. Un grand nombre d'écrits sur les coûts de transaction (Williamson 1985; Alston et Gillepsie, 1989) et les coûts de coordination (Becker et Murphy, 1992 ; Gulati et Singh, 1998, etc.) a concrétisé le projet d'analyse des dynamiques de l'intégration et des formes de gouvernance complexe auxquelles pouvaient donner lieu certains rapprochements organisationnels. On a ainsi beaucoup insisté sur les logiques de formation de réseaux d'alliances (Gulati, 1995; Moore, 1996). Le cas de la méta- 
organisation est différent puisqu'il échappe aux critères de profit et de chiffre d'affaires, en instituant des formes de contrepartie non pécuniaires, notamment par l'échange d'expériences, la mise à disposition gratuite de personnel, et la mobilisation de ressources humaines au-delà des frontières de l'organisation (Lakhani et Von Hippel, 2003). Selon la théorie de Williamson, ces éléments sont caractéristiques d'une relation d'emploi au sens où Coase pouvait la concevoir, c'est à dire permettant d'orienter les contenus de savoirs à partir de l'organisation d'une relation d'autorité. Or l'observation de la méta-organisation montre précisément qu'il y a une forme de délégation d'une telle relation de la part des personnes morales membres ou adhérents au management du secrétariat en charge de transformer l'écosystème de leurs propres entreprises ou organisation administrative. S'il existe une forme de rationalité sociale à s'engager dans une telle dynamique, celle-ci a peu à voir avec le comportement maximisateur défini par Williamson dans ses travaux. Deux auteurs (Ghoshal et Moran 1996) ont d'ailleurs insisté sur l'impasse normative à laquelle conduit une approche trop étroite du comportement des agents économiques et du marché. Comme ils le résument assez clairement dans la conclusion de leur article :

The context in which social relations and economic exchange are embedded can induce self-aggrandizement or trust, individualism or collectivism, competition or cooperation among participants. Economic progress requires both kinds of behaviors in each set of alternatives, not just one or the other. Because the logic of most markets is based on the first in each set of behavioral alternatives, organizations are necessary to protect some exchange parties from the opportunism of others so as to induce the second set of behaviors. But applying the same logic that gives rise to the need for protection does not provide the needed protection. Theories that ignore this distinction and attempt to create a model of organizations based on the logic of markets are dangerous, because the logic that creates the first set of behaviors destroys the context that is necessary for the second set. (Ghoshal et Moran 1996, p. 41)

Ainsi, en construisant des contextes propres à renouveler la confiance en diminuant les risques propres à la médiation dans l'innovation, sans mettre en place des formes de gouvernance intégrée, la méta-organisation devient un défi théorique pour les approches par les coûts de transaction. Au final, la limite du cadre néoinstitutionnaliste tient sans doute à son caractère trop statique, formel et centré sur le formel. Il se révèle ainsi peu appropriable pour saisir les dynamiques du capitalisme contemporain, qu'il s'agisse des logiques de concurrence monopolistique à l'œuvre dans la normalisation (Vion et al., 2013) ou, comme c'est le cas dans ce numéro, de l'ancrage territorial des apprentissages propres au capitalisme cognitif (Tremblay et al., 2012b; Gadille, Méhaut, Courault, 2013).

\section{Au-delà des institutions : le rôle des apprentissages territorialisés dans le capitalisme cognitif}

10 Si l'enjeu de création et de valorisation des connaissances dans le capitalisme est loin d'être nouveau, Enzo Rullani (2000) renouvelle sa place dans une définition originale du capitalisme cognitif inspirée par des travaux continus sur l'évolution de l'organisation des districts industriels. Pour cet auteur, les formes organisationnelles propres au capitalisme cognitif naissent de l'évolution du statut de la connaissance des travailleurs de plus en difficile à réduire à du capital à partir d'une relation de subordination et ce, en raison de l'incertitude croissante sur ce qui fait l'objet de l'échange dans la relation 
de travail et sa valorisation marchande. Une transformation technique importante dans l'exploitation des connaissances est liée à la diffusion des technologies de l'information et la communication, elles-mêmes ancrées dans des processus intensifiés de codification. Ceux-ci rendraient la connaissance mobilisable de manière distincte du capital et du travail employés pour la produire. Pour l'auteur, cette valorisation de la connaissance entraînerait des "mismatchings", des " espaces de crises ", d'où peuvent naître des solutions nouvelles et des transformations institutionnelles. Les métaorganisations constitueraient des espaces d'actions porteurs de nouvelles contraintes physiques (tiers-lieux), juridiques et morales facilitant la combinaison et la diffusion rapide de connaissances dans des économies où la régulation issue des figures de la grande entreprise, de l'État et des syndicats n'est plus à même de garantir l'accès à des ressources cognitives complexes pour un public plus large (PME, chômeurs, chercheurs scientifiques, etc.). Ces méta-organisations réussiraient du point de vue de l'intérêt public lorsqu'elles parviennent à réduire l'écart entre une diffusion limitée (tendant au monopole de façon intentionnelle ou pas) et une socialisation de la connaissance (y compris l'accès à l'alphabétisation pour des populations immigrées). Dans ce système, la capitalisation des connaissances et leur valorisation reposeraient sur des processus expérimentaux et différenciés par et dans des contextes singuliers. Pour Rullani, le méta-organisateur tendrait à résoudre, sans forcément y parvenir, trois sortes de mismatching (ou manque de concordance).Le premier mettrait en scène la relation entre une diffusion nécessaire pour générer de la valeur et une appropriation nécessaire pour réduire son degré de diffusion via sa socialisation. Est par exemple en jeu une régulation des standards faisant baisser de façon importante les barrières liés à la propriété sur les capitaux intellectuels (brevets, autres formes de propriété intellectuelle codifiées, connaissances tacites, etc.), tout en dessinant un processus de monopolisation plus vaste (cf. supra) et ancré dans la localité. Le deuxième mismatching serait lié au décalage entre un temps long d'apprentissage complexe inscrit dans différentes temporalités sociales («temps de la vie») et une vitesse d'apprentissage rapide propre aux temporalités de la production. D'ailleurs, dans un tel contexte, où il existe un risque de réduction de la variété et de l'indétermination jouant sur la baisse de performance de l'organisation (Tremblay 2012a). L'action publique peut contribuer à la création d'espaces de gratuité et de partages communautaires non liés directement à la création de valeur. Les méta-organisations territoriales constitueraient alors l'une des modalités de cette action publique. Le troisième mismatching découle de la relation entre le risque de se tromper (tel que perçu par les personnes, les groupes sociaux, les collectivités, les entreprises) et l'investissement cognitif dans des situations complexes où rien n'est a priori garanti. Dans ce contexte, les méta-organisations peuvent être vues comme des espaces de médiation permettant de distribuer/mutualiser les risques qui pèsent sur chacun, par des artefacts et des règles qui se voudraient partagées et équitables.

11 La création et l'organisation du secrétariat jouent ici un rôle charnière digne d'un intérêt particulier. D'une part, le secrétariat institue une coordination sociale en définissant des cadres formels et informels de collaboration; d'autre part il constitue en lui-même une communauté de pratique (au moins émergente), au sens le plus classique du terme (Orr 1990). La caractéristique de la méta-organisation ne serait-elle pas alors d'instaurer une structure organisationnelle de type communautaire articulant quatre types d'apprentissages croisés, selon les termes d'Armand Hatchuel (1996) (secrétariat/membres adhérents, secrétariat/salariés du secrétariat, membres 
adhérents/membres adhérents, salariés/salariés), matérialisant des formes d'intelligence collective à géométrie variable? Répondre à cette question impose à l'observateur d'étudier pour chaque projet ou enjeu non seulement les contours des collaborations et des engagements mutuels, mais aussi le rôle que joue l'ordre métaorganisationnel dans la transformation de ces contours en distinguant gouvernance et secrétariat. L'architecture des projets et la topologie des innovations communes sont d'une grande complexité (Gulati et al 2012), comme l'illustrent des champs aussi divers que les consortiums d'industries de défense nord-américains (Depeyre et Dumez 2010; Okros et al. 2011) ou les pôles de compétitivité français (Retour 2009 ; Boquet et al. 2009 ; Gadille et al. 2013). Si les écrits sur les communautés de pratique (Brown et Duguid 1991, Tsoukas 1996, Demazière et al. 2009) ont beaucoup insisté sur les dimensions pragmatiques de la collaboration, en insistant particulièrement sur l'importance de routines communes, ceux-ci pointent également des pratiques qui génèrent de la sélection sociale (Boudreau and Lakhani, 2011). Or cette sélection sociale est loin d'être déterritorialisée.

12 Malgré l'insistance des travaux d'économie de l'innovation sur les phénomènes de collaboration mondiale en ligne (Franke et Shah 2003, Faraj et Johnson 2011), l'enracinement territorial des acteurs pèse aussi sur leurs logiques de groupement et sur les formes de socialisation des connaissances qui soutiennent les projets communs. Cette dimension est particulièrement présente dans la politique française des pôles de compétitivité, qui fait l'objet d'une comparaison interrégionale dans la contribution de Julien Maisonnasse, Francesca Petrella et Nadine Richez-Battesti, mais on la retrouve également à l'œuvre dans le cas de la gouvernance régionale de l'innovation multimédia sur des aires métropolitaines présentées par Bruno Lusso ou des territoires de projets ruraux présentés par Dany Lapostolle.

D'un point de vue méthodologique, les cinq contributions réunies ici suggèrent l'intérêt de différentes méthodologies, voire leur complémentarité. L'heuristique de la méthodologie comparative favorisant la compréhension, à partir du général, de ce qui est particulier à la construction d'espaces d'action est largement mobilisée par les auteurs de ce numéro. Si elle est utilisée ici dans quatre cas, à des niveaux sociétaux différents, comparaison internationale (France-Italie) pour Annalisa Lendaro, et infranationale pour Bruno Lusso, Dany Lapostolle ainsi que Julien Maisonnasse et ses coauteures, le regard est toujours porté de façon approfondie sur des dynamiques d'acteurs qui (re) construisent des savoirs localisés en interaction avec des problématiques d'action publique régionalisée prenant corps par la mise en action d'un travail de méta-organisateur de réseaux (aux niveaux régionaux ou infrarégionaux).

Une autre méthode, telle que l'étude longitudinale de cas enchâssés, développée par Virginie Xhauflair, permet non seulement de comprendre finement les processus d'apprentissage collectifs débouchant sur la construction d'une méta-organisation dans un contexte sociétal (Belgique) de régulation du marché du travail, mais aussi de réfléchir directement sur le rôle délicat que peut jouer la recherche publique dans un tel processus. Ce rôle est directement lié aux méthodes que le chercheur mobilise dans la production d'effets de connaissances.

15 L'ensemble de ces textes met également en évidence des temps d'apprentissage collectifs longs et complexes, représentant des contraintes physiques, juridiques et morales que seules une collectivité, une entreprise, une association classique pourraient ou sauraient supporter. Ce constat est mis en avant dans le texte de Dany 
Lapostolle, étudiant une ingénierie territoriale qui se bureaucratise en administrant des procédures qui font des territoires de pays des segments d'exécution d'une politique définie indépendamment des enjeux territoriaux et où, malgré tout, la forme méta-organisationnelle portée par l'action citoyenne née de contingences localisées peut faire la différence. Néanmoins, la fiabilité sociale et cognitive des métaorganisations elles-mêmes reste à interroger. Leur agilité à transformer l'environnement dans la localité dépend, dans des contextes sociétaux précis, des degrés d'ouverture-fermeture sur les réseaux, des capacités de représentation des membres et de la direction, des opérations collaboratives via les compétences spécifiques des secrétariats (Gadille et Pelissier, 2009).

Pour sa part, l'article de Bruno Lusso met en évidence des différences de performances sociales et économiques importantes observées entre trois clusters multimédias français construits aux niveaux régionaux-métropolitains de la politique publique et rassemblant des entreprises, des institutions de recherche et de formation ainsi que des collectivités. Les méta-organisations des trois pôles étudiés, comprenant l'enjeu d'une hybridation des savoirs en faveur des activités transmédia, ont cherché à créer une dynamique inter-organisationnelle capable d'aller au-delà des frontières routinisées entre les filières audiovisuelles, celle du jeu vidéo, ainsi que le contenu numérique et télécommunications. Cependant, les conflits internes au pôle PRIMI à Marseille ou encore la forme de gouvernance collégiale mise en place au sein des trois associations professionnelles, cherchant à respecter scrupuleusement les grands équilibres sectoriels et à éviter tout choc éventuel témoignent d'apprentissages collectifs rugueux nécessitant du temps et des agilités dans le design de la gouvernance.

De son côté, le travail d'Annalisa Lendaro insiste plus particulièrement sur un effet sociétal produit par la conjonction d'une décentralisation à mi-chemin associée à l'intériorisation de la norme sociale et juridique de l'état colbertiste. Si l'espace de dialogue social pour des accords-cadres tripartites (État, Région, Synidcat patronal) se veut ici renouvelé, car élargi non sans réticences patronales, aux syndicats de salariés et organismes paritaires, il reste surplombé par un double impératif: celui de nondiscrimination affirmée par l'État républicain et celui d'évitement des dérives conflictuelles sur la situation des immigrés, entre partenaires de la négociation. Par contre, en Italie, où l'immigration est plus récente, cette catégorie sociale est assumée au niveau méta-organisationnel par un comité, ce qui engendre des apprentissages croisés et une performativité agissant dans un lieu plus normé de négociations.

De même, les cas étudiés par Maisonnasse, Petrella et Richez-Battesti suggèrent des similarités et des distinctions entre les logiques inter-organisationnelle et métaorganisationnelle plus proches des pôles de compétitivité par exemple. La médiation serait effective lorsque qu'un acteur tiers se positionne à l'intersection de plusieurs univers : celui des pouvoirs publics et des acteurs socio-économiques, ou encore entre ceux des acteurs socio-économiques. Pour ces auteurs, «ce positionnement serait trouvé à partir de la mise en lien d'acteurs hétérogènes en faveur d'actions collectives élargissant ou respécifiant via des débats et de la co-construction de règles communes les champs de ces acteurs. Cependant la méta-organisation constitue elle-même un espace protéiforme de la médiation, alors que dans une logique inter-organisationnelle, les espaces sont à construire».

19 C'est justement le rôle du tiers, dans cette entreprise risquée d'élaboration et de localisation d'une entité méta-organisationnelle que nous présente Virginie Xhauflair 
sous le terme de «tercéisation ", ce qui viendrait conforter et enrichir des observations antérieures (Gadille, 2008). Cette auteure insiste sur l'activité méta-réflexive dans l'apprentissage collectif et ses différentes dimensions: celle-ci ne peut-être vue uniquement sous l'angle cognitif, mais doit être abordée de manière systémique, en travaillant parallèlement et en interaction ses dimensions cognitive, politique et pragmatique : «les acteurs tiers jouent un rôle crucial dans le soutien à ces processus d'apprentissage collectif, en endossant une fonction de tercéisation qui met les partenaires des projets innovants en capacité de travailler les différentes dimensions de leur réflexivité ».

\section{Le méta-organisateur comme " maître de contexte » fragile}

Dans le cas de la méta-organisation territoriale, qu'il s'agisse ici des régions françaises ou italiennes, des communautés belges, ou des territoires intermédiaires de type "pays", les entreprises d'intermédiation jouent donc un rôle cognitif dans la génération, l'exploitation et la diffusion de la connaissance.

21 L'intermédiarité peut être définie comme une situation particulière où l'autorité est redistribuée et les hiérarchies affaiblies au profit d'une cogestion des affaires publiques entre un nombre de plus en plus élevé d'acteurs publics et privés (Duran, 1999). De telles dynamiques intermédiaires sont au cœur des recompositions de l'action publique (Filâtre et Terssac, 2005) et des dynamiques locales d'innovation (Tremblay, 2012b), car elles reconstruisent la nature de l'autonomie et du contrôle dans les collectifs, ces dynamiques s'organisant alors autour de l'émergence de nouvelles catégories sociales, mobilisant territoire et dispositif. Généralement, si les travaux sur la gouvernance mettent en évidence des processus d'apprentissage synonymes de transformation de règles, ils soulignent en même temps l'hétérogénéité de ceux-ci, ainsi que leur instabilité et leur incomplétude, selon les objets de la politique publique. Les nouveaux dispositifs de réforme et d'action de l'État, tels que celui des pôles de compétitivité en France, semblent alors s'inscrire dans la formation d'une « méta-gouvernance » définie sur la base des théories inter-organisationnelles comme " un concept englobant qui modélise le rôle de l'État et ses instruments politiques caractéristiques dans la gouvernance de réseaux» (Jessop, 2007, p. 541). Dans ces approches, la notion de territoire possède une dimension sociétale : le territoire est conçu comme un système d'acteurs porteurs de projets. Ces considérations invitent à une approche cognitive, politique et technique de l'intermédiation dans la transformation de normes et structures sociales. Elles posent la question des conditions de création de «nouveaux espaces de connaissances " (Hatchuel, 2000) et celle des logiques d'internalisation par les organisations de leurs rapports à la société et aux formes d'autorité qui la traversent (Maurice, 2008). La manière dont Annalisa Lendaro rend compte, dans ce numéro, de l'internalisation différenciée des rapports à l'immigration de travail selon les sociétés, ainsi que des différentes formes d'intermédiation régionales auxquelles donne lieu cette internalisation, ouvre une voie comparative féconde.

Plus généralement, la réflexion sur la notion d'intermédiaire nous conduit à mieux comprendre ce qui advient d'un point de vue cognitif dans le travail de métaorganisateur et par conséquent dans l'organisation et les pratiques de gestion de ces intermédiaires. 

organisateur " dans les réseaux marchands, connectés aux réseaux sémantiques et sociaux (Rulliani, 2000, p. 105). Il construit de nouvelles institutions qui permettent de gouverner les trois types de conflits liés à l'accès à la connaissance et aux réseaux, à la relation entre vie et travail et enfin à la distribution sociale du risque. "Le métaorganisateur est le pouvoir émergeant de nécessités fonctionnelles de relations qui doivent coordonner beaucoup d'opérateurs et beaucoup d'intérêts différents » (Ibid., p. 104). Ce que les études de Virginie Xhauflair et Julien Maisonnasse et al. mettent en avant est que cette capacité de consolidation de nécessités fonctionnelles reste fortement contingente et assortie à des logiques d'intermédiation légitimées par l'investissement actif de tiers agissant tant sur les dimensions cognitive et politique que sur une méta-réflexivité. De ce point de vue, le processus d'intermédiation et le processus de "tercéisation » apparaissent comme la condition sociale de la conception et de la co-construction du nouveau modèle d'organisation.

Par ailleurs, si ces méta-organisateurs se construisent dans la continuité de la stratification salariale des entreprises ou des administrations publiques, ils ne constituent pas moins à partir de leur position politique et projective, un attracteur différencié sur et dans la construction des marchés du travail. Leurs savoirs et savoirfaire reconnus sont le résultat de bifurcations et d'hybridations qui ont fait émerger des compétences singulières. Dans le cas présenté par Virginie Xhauflair la création d'une forme triangulaire d'emploi à partir de la matérialisation d'un groupement d'employeurs a impliqué que les membres de ce groupement apprennent ensemble dans le design et l'instauration d'une structure de gouvernance et en même temps acceptent des risques liés au transfert de certaines de leurs prérogatives à cette même structure. Il résulte de ces mobilités vers les méta-organisateurs des combinatoires de savoirs plus ou moins novatrices et porteuses de capacités d'innovation sociale dans les territoires. Cela a deux conséquences pour la gouvernance territoriale : la première est que le méta-organisateur, de par sa position centrale, maîtrise le contexte local de la méta-organisation, qu'il lui est loisible de cadrer, et que les organisations parties prenantes peuvent le cas échéant déborder, au prix de déloyautés partielles ou de mise en concurrence des méta-organisations ; la seconde est que la position de métaorganisateur territorial aboutit parfois à la mobilisation de compétences proches de celles des acteurs politiques traditionnels, en ce qu'elle devient, dans des espaces interprofessionnels complexes, une "compétence du général » (Mayntz, 1983). Cette compétence semble toutefois être moins centrée sur la mobilisation d'expertises individuelles que sur celle de connaissances distribuées et validées par des collectifs dans des espaces émergents en voie d'institutionnalisation ; elle est également plus intégrative que celle de certaines entreprises ou organismes professionnels investis dans des activités de conseils, consultance ou rôle d'observatoire.

Ces deux éléments doivent nous conduire à insister sur l'équilibre instable des métaorganisations territoriales du fait de zones de vulnérabilité identifiées par Göran Arnhe et Nils Brunsson (2010) : l'augmentation du nombre de membres rend difficile le maintien du consensus, et la concurrence interne sur le contrôle des activités, qui s'opère entre les niveaux de l'organisation et du secrétariat, peut augmenter au point de provoquer des crises. Le plus souvent, l'alternative qui en résulte est donc soit une intégration verticale plus poussée par souci d'économie des coûts de coordination, soit les stratégies d'exit de certains membres dotés de ressources capitales. La fragilité de 
cet équilibre confronte donc la méta-organisation à une difficulté, qui est celle de se renouveler en maintenant un équilibre entre règles formelles et informelles (Gulati et Puranam, 2009). Les cas présentés par Dany Lapostolle montrent que la résolution des problèmes par la formalisation aboutit le plus souvent à une forme d'intégration bureaucratique qui rigidifie les relations et asphyxie les dynamiques de projet à compléter. Le contraste avec le cas régional italien dans l'étude d'Annalisa Lendaro est ici frappant. Elle montre, dans ce cas, que la construction d'une catégorie d'immigré performative prend forme dans l'interaction entre un espace de concertation plus ouvert et transversal et donc moins normatif (le Comité élargi à la société civile) qui joue ici un rôle de méta-organisation, et un espace de régulation de branche plus normé et restreint aux partenaires sociaux, mais bénéficiant des apprentissages collectifs du premier.

Chaque article, en présentant une description soignée de ces dispositifs et de leur contexte d'émergence vient conforter une approche « orientée phénomène » en théorie des organisations et en même temps réactualise la question du "design " physique et organisationnel dans ce champ (Pfeffer, 1997, p. 196). Un cadre comparatif plus global, qui prendrait en compte les effets croisés de la construction de la méta-organisation territoriale avec les dynamiques sectorielles et locales offrirait un regard renouvelé des variétés de capitalisme à partir d'une compréhension plus fine de ce phénomène. Le rôle de facilitation des acteurs publics mis en avant dans les études de ce numéro est-il une propriété des capitalismes dits coordonnés ou une réalité beaucoup plus globale? Les méta-organisations territoriales renforcent-elles d'autres dynamiques métaorganisationnelles comme celles de la branche, ou les fragilisent-elles au contraire ? La multiplication de ces structures est-elle un signe de transformation en profondeur de cohérences entre systèmes (industriel, éducatif, etc.) qui se modifient ailleurs autrement ou est-elle seulement un révélateur de difficultés récurrentes de transformations socio-économiques, mais aussi juridiques ? Les formes de standardisation mise en place dans les territoires sont-elles solubles dans les dynamiques plus globales de la standardisation (Brunsson et Jacobson, 2000), ou sontelles seulement génératrices de franges locales? Ces questions ne trouvent pas de réponse immédiate dans le numéro, mais invitent à structurer un programme de recherche sur la base de collaborations entre centres de recherche. Si ce numéro est une pierre de plus dans une telle entreprise, nous nous en réjouirons.

\section{BIBLIOGRAPHIE}

Aggeri, Franck., Le Masson, P., Branciard, Anne., Paradeise, Catherine., \& Peerbaye, A. (2007). Les plates-formes technologiques dans les sciences de la vie. Politiques publiques, organisations et performances. Revue d'économie industrielle, (120), 21-40.

Alston, Lee J., and William Gillespie (1989). "Resource coordination and transaction costs." Journal of Economic Behavior and Organization, 11: 191-212.

Arhne, Göran, et Nils Brunsson, (2010). « How much do meta-organizations affect their members? ", 7th Pan-European International Relations Conference, Stockholm, 9-11 September. 
Arhne, Göran et Nils Brunsson (2004), "Soft Regulation from an organizational perspective”, In Ulrika Mörth (ed), Soft Law in Governance and Regulation - An Interdisciplinary Analysis, Cheltenham: Edward Elgar

Bagnasco, Arnaldo (1977). Tre lialia: la problematica territoriale dello svilluppo, Bologne, Il Mulino.

Becker, Gary S. et Murphy, Kevin M. (1992). The division of labor, coordination costs, and knowledge. The Quarterly Journal of Economics, vol. 107, no 4, p. 1137-1160.

Benko, George et Dunford, Mick eds. (1991). Industrial Change and Regional Development, Londres, Pinter/Belhaven Press, 329 pages.

Boquet, Rachel, Mendez, Ariel, et Mothe, Caroline (2009), Pôles de compétitivité constitués de PME: quelle gouvernance pour quelle performance?. Management \& Avenir, $n^{\circ}$ 5, p. 227-244.

Brail, Shauna et Gertler, Meric S. (1999). The digital regional economy. Emergence and evolution of Toronto's multimedia cluster, in: Hans-Joachim Braczyk, , Georg Fuchs et Hans-Georg Wolf (Eds) Multimedia and Regional Economic Restructuring (London: Routledge).

Brenner, Thomas (2005). Innovation and cooperation during the emergence of local industrial clusters: An empirical study in Germany. European Planning Studies, vol. 13, n 6, pp. 921-938.

Britton, J., Tremblay, Diane-Gabrielle et Smith, R. (2009). Contrasts in clustering: the example of Canadian new media, European Planning Studies, vol.17, n², pp. 211-235.

Brown, John Seely et Duguid, Paul(1991). Organizational learning and communities-of-practice: Toward a unified view of working, learning, and innovation. Organization science, vol. 2, no 1, pp. 40-57.

Brusco, Sergio, (1982), The Emilian model: productive decentralisation and social integration, Cambridge Journal of Economics, 6,167-184.

Burt Ronald (1992), Structural holes. The social structures of competition, Harvard University Press, 324 pages.

Callon, Michel, (1986). « Éléments pour une sociologie de la traduction. La domestication des coquilles Saint-Jacques dans la Baie de Saint-Brieuc », dans L'Année sociologique, n³6.

Coase, Ronald, H. (1937) "The nature of the firm." Re- printed in G. J. Stigler and K. E. Boulding (eds.), A.E.A. Readings in Price Theory: 331-351.

Conein, Bernard. (2004), Communautés épistémiques et réseaux cognitifs: coopération et cognition distribuée. Revue d'économie politique, vol. 113, p. 141-159.

Delgado, Mercedes, Porter, Michael E., et Stern, Scott. (2010) Clusters and entrepreneurship. Journal of Economic Geography, 2010, vol. 10, no 4, p. 495-518.

Demazière, Didier, Horn, François et Zune, Marc (2009). « La socialisation dans les « communautés » de développement de logiciels libres », Sociologie et Sociétés, Vol. 40, n², p. 217-238.

Depeyre, Colette, Dumez, Hervé, et al. (2010), The role of architectural players in coopetition: The case of the US defense industry. Coopetition: winning strategies for the 21st century. Edward Elgar, Cheltenham, p. 124-140.

Dudouet, François-Xavier, Mercier, Delphine, et Vion, Antoine. (2006) Politiques internationales de normalisation. Revue française de science politique, vol. 56, no 3, p. 367-392.

Duran, Patrice (1999), Penser l'action publique, Paris, LGDJ, 212 pages. 
Ehlinger, Sylvie, Perret, Véronique, et Chabaud, Didier. Quelle gouvernance pour les réseaux territorialisés d'organisations?. Revue française de gestion, 2007, no 1, p. 155-171.

Evans, Philip, et Wolf, Bob (2005). Collaboration rules. Harvard Business Review 83(7/8): 96-104.

Faraj, Samer, et Steven L. Johnson (2011). Network exchange patterns in online communities. Organization Science 22(5): 1464-1480. 37

Filatre, Daniel et Gilbert de Terssac (eds.) (2005), Les dynamiques intermédiaires au cœur de l'action publique, Toulouse, Octares.

Franke, Nicolaus et Sonali K. Shah 2003. How communities support innovative activities: an exploration of assistance and sharing among end-users. Research Policy 32(1): 157-178.

Gadille, Martine, (2008), « Le tiers comme agent de réflexivité et accélérateur d'apprentissages collectifs : le cas du dispositif des pôles de compétitivité », n²89, p. 61-79.

Gadille, Martine et Pélissier, Maud (2009). «Les PME multimédia et logiciel éditeur dans le pôle de compétitivité 'Solutions Communicantes Sécurisées' : Quel mode de gouvernance pour quelle intégration industrielle?», Management et Avenir, n² 25, pp. 207-226.

Gadille, Martine, Méhaut, Philippe, et Courault, Bruno (2013). Compétences et régulation des marchés du travail dans les pôles de compétitivité: le cas du pôle Pégase. Revue d'Économie Régionale \& Urbaine, $\mathrm{n}^{\circ}$ 2, pp. 339-361.

Gaudin, Jean-Pierre (1999). Gouverner par contrat : l'action publique en question, Paris, Presse de Sciences Po, 233 pages.

Ghoshal, Sumantra et Moran, Peter. Bad for practice: A critique of the transaction cost theory. Academy of management Review, 1996, vol. 21, no 1, p. 13-47.

Gibbons, Michael, Limoges, Camille, Nowotny, Helga, Schwartzman, Simon, Scott, Peteret Trow, Martin (1994).The new production of knowledge: The dynamics of science and research in contemporary societies. Sage, 192 pages.

Glaeser, Edward L., Kerr, William R., et Ponzetto, Giacomo A M. (2010), Clusters of entrepreneurship. Journal of Urban Economics, vol. 67, $\mathrm{n}^{\circ}$ 1, p. 150-168.

Gulati, Ranjay, et Phanish Puranam (2009). Renewal through reorganization: the value of inconsistencies between formal and informal organization. Organization Science vol.20, $\mathrm{n}^{\circ} 2$, pp. 422-440.

Gulati Ranjay, Singh H. 1998. The architecture of cooperation: managing coordination costs and appropriation concerns in strategic alliances. Administrative Science Quarterly, vol 43, nº 4 pp. 781-814.

Gulati Ranjay (1995). Social structure and alliance formation patterns: a longitudinal analysis. Administrative Science Quarterly, vol. 40, n, pp. 619-652.

Gulati Ranjay, Puranam Phanish, et Michael Tushman. (2012): "Meta-Organizational Design: Rethinking Design in Inter-Organizational and Community Contexts." In Special Issue on Strategy and the Design of Organizational Architecture edited by Ranjay Gulati, Phanish Puranam, Michael Tushman, Strategic Management Journal, vol. 33, $n^{\circ} 6$ pp. 571-586.

Hatchuel, Armand, (1996), « Coopération et conception collective. Variété et crises des rapports de prescription. In de Terssac, Gilbert, Friedberg Eyrhard. (coord.) Coopération et conception. Toulouse : Octares, pp. 101-122. 
Hatchuel, Armand., (2000), Prospective et gouvernance : quelle théorie pour l'action collective? dans : Prospective pour une gouvernance démocratique, colloque de Cerisy, coord. Elisabeth. Heurgon et José Landrieu, pp . 29-41.

Jessop, Bob (1999). The dynamics of Partnerships and Governance Failure. In G. Stocker (ed.), The new Politic of Local Governance in Britains, Oxford: Oxford University Press, $10 \mathrm{p}$.

Jessop, Bob (2007) Governance and metagovernance: on reflexivity, requisite variety, and requisite irony'. In M. Bevir. Ed., Public governance. Volume 1. Theories ofgovernance. London: Sage, pp. 230-45.

Lakhani, Karim R, von Hippel, Eric (2003). How open source software works: "free" user-to-user assistance. Research Policy 32(6): 923-943.

Latour, Bruno, (2001), L'espoir de Pandore. Pour une version réaliste de l'activité scientifique » Paris : La Découverte, Paris, 347 p.

Lawrence, Paul R. et Jay W. Lorsch (1967). Organization and Environment: Managing Differentiation and Integration. R.D. Irwin: Homewood, IL.279 pages

Lazega, Emmanuel (2009), "Théorie de la coopération entre concurrents : Organisation, marché et analyse de réseaux", in Philippe Steiner et François Vatin (eds), Traité de sociologie économique, Paris, Presses Universitaires de France, 816 pages

Le Bianic, Thomas et Vion, Antoine (2008). Action publique et légitimité professionnelle, Paris, LGDJ, 348 pages

Lemarie, Stéphane, Mangematin, Vincent et Torre, André (2001). Is the creation and development of biotech SMEs localised? Conclusions drawn from the French case. Small Business Economics, vol. 17, no 1-2, p. 61-76.

Maillard (de), Jacques (2004). Réformer l'action publique. La politique de la ville et les banlieues, Paris, LGDJ, 242 pages.

Mauric, Marc, (2008). La construction sociale des acteurs de l'entreprise, Toulouse, Octarès.

Mayntz, Renate (1983). The Conditions of Effective Public Policy - A New Challenge for Policy Analysis", Policy and Politics 11, 2, pp. 123-143.

Moore, James F. (1996). The Death of Competition: Leadership and Strategy in the Age of Business Ecosystems. Harper Business: New York, NY, 297 pages.

Okros, Alan, Verdun, John et Chouinard, Paul (2011). The Meta-Organization.A Research and Conceptual Landscape, Defence R\&D Canada - Centre for Security Science, DRDC CSS TR 2011-13, July, 74 pages.

Orr, Julian E. (1990). Sharing knowledge, celebrating identity: Community memory in a service culture. Collective remembering, vol. 169, pp. 189.

Paraponaris, Claude, Sigal Martine, Vion, Antoine (2013) « Communautés et collectifs : des dynamiques différentes pour la génération des connaissances », Revue internationale de psychosociologie des comportements organisationnels, à paraître.

Pfeffer Jeffrey (1997). New directions for organization theory. Problems and prospects. Oxford University Press, New York, 264 pages.

Pfeffer Jeffrey, Salancik Gerald R. (1978). The External Control of Organizations: A Resource Dependence Perspective. Harper \& Row: New York, NY. 300 pages 
Pyke Frank, Sengenberger Werner (1992) (Eds) Industrial Districts and Local Economic Regeneration, International Institute for Labour Studies, Geneva, 294 pages.

Retour, Didier. (2009) Pôles de compétitivité, propos d'étape. Revue française de gestion, $\mathrm{n}^{\circ}$ 10, pp. 93-99.

Robertson, Paul L. (2006). Transaction Costs, Trust, and the Structuring of Markets. Discussion Paper No. 342, October, School of Economics, The University of Queensland, Australia, https:// www.google.fr $/ \# \mathrm{q}=$ Transaction+Costs $\% 2 \mathrm{C}+$ Trust $\% 2 \mathrm{C}+\mathrm{and}+$ the+Structuring+of+Markets+$+\mathrm{UQ}+\mathrm{eSpace}, 12$ novembre.

Robinson, Douglas KR, Rip, Arie, et Mangematin, Vincent. Technological agglomeration and the emergence of clusters and networks in nanotechnology.Research policy, 2007, vol. 36, no 6, p. 871-879.

Rullani, Enzo (2013). Territori in transizione: nuove reti e nuove identità per le economie e le società locali sinergie, rivista di studi e ricerche, n 91, Maggio-Agosto 2013, pp. 141-163.

Rullani, Enzo (2000). Production de connaissance et valeur dans le postfordisme, entretien avec Enzo Rulliani, par Antonella Corsani, multitudes, 2, mai, pp. 97-110.

Steyer, Alexandre, Deroïan, Fredéric, Zimmermann, Jean-Benoît (2003). Apprentissage social et diffusion de l'innovation : réseaux critiques et intermédiarité, Revue d'économie industrielle, $\mathrm{n}^{\circ} 103$, pp. 71-89.

Tremblay, Diane-Gabrielle (2012a) Performance organisationnelle et temps sociaux. Presses de l'Université du Québec.

Tremblay, Diane-Gabrielle, Juan-Luis Klein, Angélo Dossou-Yovo et Tarek Ben Hassen (2012b) Les acteurs intermédiaires dans le développement de l'innovation: une comparaison intersectorielle dans la région de Montréal. Revue d'Économie Régionale \& Urbaine, n³, p. 431-454.

Tsoukas, Haridimos (1996). « The firm as a distributed knowledge system », Strategic Management Journal, Winter, Special Issue, $\mathrm{n}^{\circ}$ 17, pp. 11-25.

Vion Antoine., Diaz Pablo A., Dudouet François-Xavier et Graz Jean-Christophe (2013).

« Normalisation informatique et marchés de services. Le cas du XML ». In Graz Jean.-Christophe, Niang Nafi., Services sans frontières?, Paris : Presses de Sciences Po, Coll. Gouvernances.

Weber, Max (2000) [1894]. 'Stock and Commodity Exchanges' [Die Börse], Theory \& Society, $\mathrm{n}^{\circ} 29$, pp. 305-338.

Williamson, Oliver E. (1985). The Economic Institutions of Capitalism. New York: Free Press. 1991 "Comparative economic organization: The analysis of discrete structural alternatives", Administrative Science Quarterly, n³6, pp. 269-296.

Williamson, Oliver E. (1983). Markets and Hierarchies: Analysis and Antitrust Implications. New York: Free Press, 286 pages.

Zeller, Christian (2001). Clustering biotech: a recipe for success? Spatial patterns of growth of biotechnology in Munich, Rhineland and Hamburg, Small Business Economics, n 17, pp. 123 -141.

\section{NOTES}

1. Traduit par nous-mêmes 


\section{AUTEURS}

MARTINE GADILLE

Aix Marseille Université, CNRS, LEST UMR 7317, 13626, Aix en Provence, France

\section{DIANE-GABRIELLE TREMBLAY}

Professeure, École des sciences de l'administration, TÉLUQ | Université du Québec

\section{ANTOINE VION}

Aix Marseille Université, CNRS, LEST UMR 7317, 13626, Aix en Provence, France 